for patients with chronic disease. Numbers of completed plans are low, partly because some patients do not wish to complete one, and partly due to practical barriers to completion.

Aim To overcome practical barriers to completion of advance care plans.

Methods From April 2011 our Care Coordination Service established a fortnightly home- based Advance Care Planning Clinic, in which two staff visit patients at their home. In August 2012 this was supplemented by a fortnightly hospital-based clinic. Patients can be booked into either clinic. In the clinic, an Advance Care Plan is prepared comprising of a Statement of Choices and may include Enduring Power of Attorney, witnessed by an authorised clinic staff member. Partners are also offered the opportunity to complete their advance care plan at this time.

Results In January-March 2011, prior to the commencement of the clinic, there were 54 patients receiving care coordination of whom 19 (35\%) had a completed advance care plan, including a Statement of Choices. In October-December 2012 there were 89 patients who had received care coordination of whom 35 (39\%) had a completed advance care plan.

Conclusion The establishment of Advance Care Planning Clinics has been a practical way to help overcome multifactorial barriers to preparing a Statement of Choices, providing increased access opportunities and support for timely completion. This approach enabled staff to improve completion rates whilst managing a $65 \%$ caseload increase.

\title{
Posters
}

\section{IMPACT OF AN ADVANCE CARE PLANNING CLINIC ON PATIENTS OF A REGIONAL CARE COORDINATION SERVICE}

J Ironside, ${ }^{1}$ W Appleton, ${ }^{1}$ P Dugdale ${ }^{1,2}{ }^{1}$ Division of Medicine, Canberra Hospital and Health Services (CHHS); ${ }^{2}$ Centre for Health Stewardship, Australian National University

10.1136/bmjspcare-2013-000491.136

Background CHHS policy calls for staff to complete advance care plans (including a statement of choices) 